\title{
Initial isolated Takayasu's arteritis of bilateral pulmonary artery branches with severe pulmonary hypertension: a case report.
}

\author{
Zhu Gangjie, Yang Jianmin*, Xu Peng \\ Department of Cardiovasology, Hangzhou First People Hospital (Affiliated Hangzhou Hospital of Nanjing Medical \\ University), No.261, Huansha Road, Hangzhou City, PR China
}

\begin{abstract}
Background: Takayasu's arteritis is a chronic systematic inflammatory disease. Isolated TA of pulmonary artery branches is very rare.

Methods: The patient received some laboratory tests (including blood routine examination, erythrocyte sedimentation rate, C-reactive protein, etc.) and imageological examinations (including echocardiography, computed tomographic pulmonary angiography, computed tomographic aortic angiography, right-sided heart catheterization, ${ }^{18}$ F-FDG PET, etc.) for diagnosis.

Results: Computed Tomographic Pulmonary Angiography (CTPA) and Right-sided Heart Catheterization (RHC) showed diffused stenosis in bilateral pulmonary arteries in the case. Pulmonary Artery Systolic Pressure (PASP) measured by echocardiography is 89 mmHg. Besides, an ${ }^{18}$ F-FDG PET showed the pulmonary arteries and the right ventricle uptake was high.

Conclusion: The symptoms of this patient have been controlled with the treatment of drugs, but pulmonary hypertension is not improved. Further treatment would be a great challenge.
\end{abstract}

Keywords: Takayasu's arteritis (TA), Pulmonary hypertension, Large vessel vasculitis. Abbreviations: CT: Computed Tomography; CTPA: Computed Tomographic Pulmonary Angiography; ESR: Erythrocyte Sedimentation Rate; FDGPET: ${ }^{18}$ F-Fluorodeoxyglucose Positron Emission Tomography; PASP: Pulmonary Artery Systolic Pressure; RHC: Right-sided Heart Catheterization; TA: Takayasu's Arteritis.

Accepted on June 5, 2018

\section{Introduction}

Takayasu's Arteritis (TA) is a chronic systematic inflammatory disease, which easily affects aorta and its major branches, including aortic arch and its branches, descending aorta, renal artery, etc. [1]. As aortic secondary branch, pulmonary artery is often involved, but isolated TA of pulmonary artery is very rare. We will report a case of Initial isolated Takayasu's arteritis of bilateral pulmonary artery branches with severe pulmonary hypertension.

\section{Case Description}

A 35 years old Chinese woman was presented to the cardiovascular disease clinic with a 7 months history of chest tightness and palpitation after activities. During these 7 months, cough and sputum occurred occasionally and antibiotics were effective. However, chest tightness and palpitation after activities did not improve. And besides, the patient was healthy previously without medical history and relevant family history.

On examination, temperature was $36.7^{\circ} \mathrm{C}$, blood pressure $121 / 76 \mathrm{mmHg}$ in the right arm and $116 / 82 \mathrm{mmHg}$ in the left, pulse 86 beats per min and room air saturation $98 \%$. Radial and dorsalis pedis artery pulses were equal and palpable bilaterally. There was a systolic murmur in pulmonary valves area. The rest of her physical examination was unremarkable.

On laboratory tests, white blood cells were $11.2 \times 10^{9} / \mathrm{L}, \mathrm{C}$ reactive protein $1 \mathrm{mg} / \mathrm{L}$, Erythrocyte Sedimentation Rate (ESR) $14 \mathrm{~mm} / \mathrm{h}$. Platelet count, hemoglobin, levels of electrolytes, and renal and liver function were normal.

Furthermore, an echocardiography showed right heart dilation and pulmonary hypertension (Pulmonary Artery Systolic Pressure, PASP $89 \mathrm{mmHg}$ ) and a lung Computed Tomography (CT) was suggestive of pulmonary infraction in middle lobe of right lung and inferior lobe of left lung. A Computed Tomographic Pulmonary Angiography (CTPA) showed varying degrees of stenosis and occlusion in bilateral lobar and segmental arteries (Figure 1). Right-sided Heart Catheterization (RHC) confirmed diffuse stenosis in bilateral pulmonary arteries. We monitored pressures in the distal left pulmonary artery, the proximal left pulmonary artery, the right ventricle, the right atrium and the superior vena cava, which were $24 / 9 \mathrm{mmHg}, 89 / 17 \mathrm{mmHg}, 100 / 4 \mathrm{mmHg}, 9 / 5 \mathrm{mmHg}$ and $10 / 6 \mathrm{mmHg}$ separately by RHC. We also noticed no pressure gradient between the right ventricle and the pulmonary trunk. Besides there was no obvious stenosis in the aortic arch and its 
branches, the thoracic aorta abdominal aorta, renal arteries, the iliac arteries and the femoral arteries in an examination of computed tomographic aortic angiography (Figure 2). The patient accepted an ${ }^{18} \mathrm{~F}-\mathrm{FDG}$ PET, which showed the pulmonary arteries and the right ventricle uptake was high.

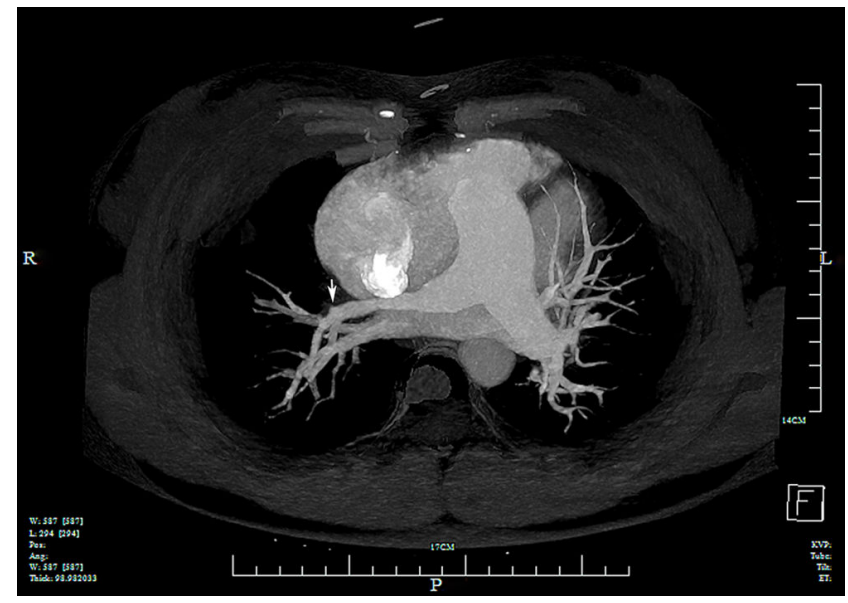

Figure 1. Total occlusion in the right upper lobar (showed by an arrow), and varying degrees of stenosis in bilateral lobar and segmental arteries.

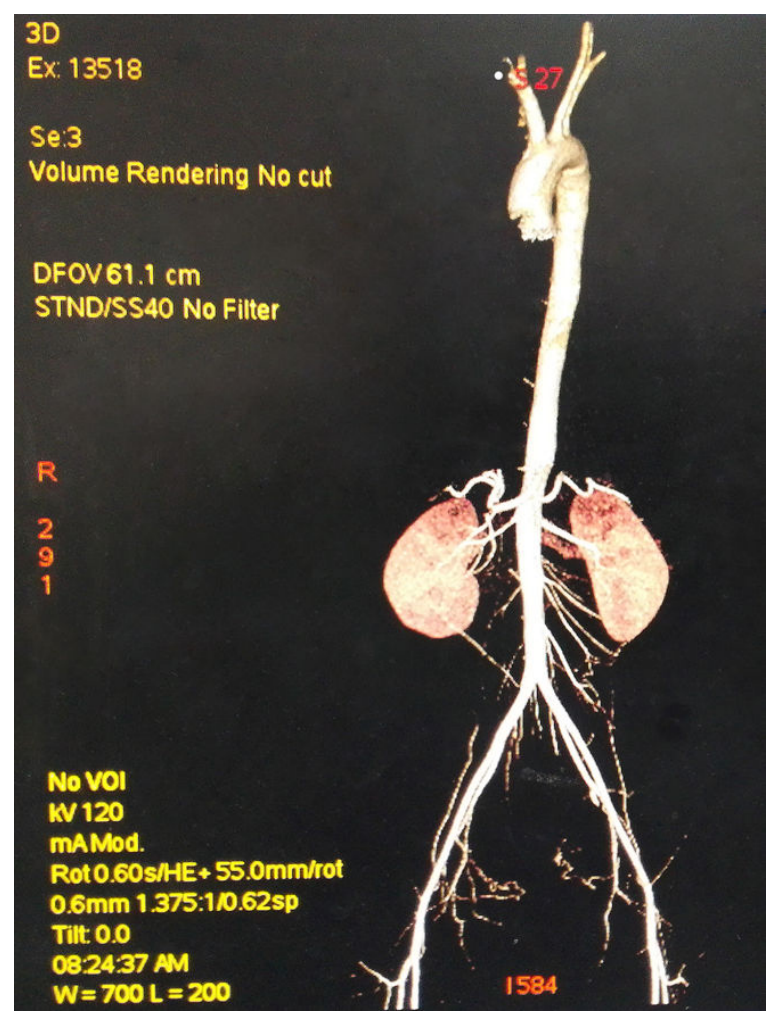

Figure 2. No obvious stenosis in the aortic arch and its branches, the thoracic aorta abdominal aorta, renal arteries, the iliac arteries and the femoral arteries.

Drugs therapy was chosen for this patient to ameliorate of her symptoms and improve pulmonary hypertension. The treatment program included four parts of drugs, which were for pulmonary hypertension (beraprost sodium $120 \mu \mathrm{g}$ daily for three times, which is a kind of prostaglandins and bosentan 125 mg daily for two times, which is a kind of endothelial receptor antagonists), for heart failure (furosemid $20 \mathrm{mg}$ daily, which a kind of loop diuretics), for thrombus (aspirin $0.1 \mathrm{~g}$ daily, which is a kind of antiplatelet agents) and for TA (prednisone $60 \mathrm{mg}$ daily with tapering weekly, which is a kind of glucocorticoids and cytoxan $400 \mathrm{mg}$ weekly for $4 \mathrm{w}$, which is a kind of cytotoxic agents). The patient has been followed up in our clinic for one and a half year. The symptoms have been improved, but recent echocardiographies showed that PASP still sustained $90 \mathrm{mmHg}$ approximately.

\section{Discussion}

Takayasu's arteritis is a rare disease of unknown etiology which occurs more often in women under the age of 40 years; it is characterized by medium and large vessel vasculitis [1]. Isolated TA of pulmonary artery is very rare and easy to misdiagnose [2]. The bases of our diagnosis with TA in this patient are: 1 . young woman, $<40$ y old; 2 . diffuse stenosis in bilateral pulmonary arteries; 3 . suggestion of ${ }^{18}$ F-FDG PET $[3,4]$; 4 . no evidence of other vasculitis.

Obviously, pulmonary hypertension of this patient is caused by pulmonary arteritis. Generally, pulmonary artery reconstruction (including balloon dilatation, stenting and bypass) can be used to improve pulmonary hypertension and patient's symptoms $[2,5]$. But for this patient, pulmonary artery reconstruction doesn't apply to the diffuse stenosis in bilateral pulmonary arteries. There was no cure for TA. Improvement of symptoms and delaying progression of TA are the most important targets of treatment. For TA with pulmonary hypertension, the targets of treatment are to reduce pulmonary pressure, control right heart failure, delay progression of TA and improve patient's prognosis. Some kinds of drugs are suggested to be used for pulmonary hypertension, including prostaglandins, endothelin receptor antagonists, guanylate cyclase agonists, and type 5 phosphodiesterase inhibitor [2,6]. If feasible, pulmonary artery reconstruction is an option [5]. For another, treatment of TA is even more important, which includes glucocorticoids and immunosuppressants [1,7]. Glucocorticoids in combination with immunosupperessants should been considered for refractory TA. For this patient, prednisone combining with cytoxan has been used for controlling TA. By tapering, dose of prednisone has been reduced from $60 \mathrm{mg}$ to $5 \mathrm{mg}$ daily.

The symptoms of this patient have been controlled with the treatment of drugs, but pulmonary hypertension is not improved, and her PASP is still $90 \mathrm{mmHg}$ approximately. If pulmonary hypertension of the patient worsens, we will have no effective treatment in the condition of pulmonary artery reconstruction to be inapplicable. Recently, biological agents, such as tumor necrosis factor-alpha inhibitors, interleukin-6 inhibitor, tocilizumab and abatacept, maybe an optional therapy $[8,9]$. But more randomized controlled trials are still needed to verify their effectiveness. Moreover, lung transplantation, as the last resorts, maybe effective in this patient [6]. However, there is no report about lung transplantation to treat TA with severe pulmonary hypertension currently in the world. 


\section{Informed Consent}

Informed consent was obtained from the patient for publication of this case report and any accompanying images. The study was accepted by the Ethics Committee of the hospital and they encouraged publishing the article (Chinese scientific medical ethic review No.2017-108-01).

\section{References}

1. Maffei S, Di Renzo M, Bova G, Auteri A, Pasqui AL. Takayasus arteritis: a review of the literature. Intern Emerg Med 2006; 1: 105-112.

2. Wang X, Dang A, Chen B, Lv N, Liu Q. Takayasu arteritis-associated pulmonary hypertension. J Rheumatol 2015; 42: 495-503.

3. Alibaz-Oner F, Dede F, Ones T, Turoglu HT, Direskeneli H. Patients with Takayasus arteritis having persistent acute-phase response usually have an increased major vessel uptake by 18F-FDG-PET/CT. Mod Rheumatol 2015; 25: 752-755.

4. Chrapko BE, Chrapko M, Nocuń A, Stefaniak B, Zubilewicz T, Drop A. Role of 18F-FDG PET/CT in the diagnosis of inflammatory and infectious vascular disease. Nucl Med Rev Cent East Eur 2016; 19: 28-36.

5. Qin L, Hong-Liang Z, Zhi-Hong L, Chang-Ming X, XinHai N. Percutaneous transluminal angioplasty and stenting for pulmonary stenosis due to Takayasus arteritis: clinical outcome and four-year follow-up. Clin Cardiol 2009; 32: 639-643.
6. Galie N, Humbert M, Vachiery JL, Gibbs S, Lang I, Torbicki A, Simonneau G, Peacock A, Vonk Noordegraaf A, Beghetti M, Ghofrani A, Gomez Sanchez MA, Hansmann G, Klepetko W, Lancellotti P, Matucci M, McDonagh T, Pierard LA, Trindade PT, Zompatori M, Hoeper M. 2015 ESC/ERS guidelines for the diagnosis and treatment of pulmonary hypertension. Rev Esp Cardiol (Engl Ed) 2016; 69: 177.

7. Ueno M. Antiplatelet therapy in the treatment of Takayasu arteritis. Circ J 2010; 74: 1079-1080.

8. Szabo MZ, Kiss E. Recent advances in the treatment of large vessel vasculitides. Orv Hetil 2017; 158: 5-12.

9. Ferrante A, Ciccia F, Guggino G, Colomba D, Triolo G. Tocilizumab therapy for unresponsive pulmonary arterial hypertension in a patient with Takayasu arteritis. Scand J Rheumatol 2016; 45: 251-252.

\section{*Correspondence to}

Yang Jianmin

Department of Cardiovasology

Hangzhou First People Hospital

Affiliated Hangzhou Hospital of Nanjing Medical University

PR China 